\title{
GW23-e0648 THE EFFECT OF MACROPHAGES ON THE BIOLOGICAL FUNCTIONS OF EPCS UNDER HIGH GLUCOSE CONDITIONS AND ITS POSSIBLE MECHANISM
}

doi:10.1136/heartjnl-2012-302920a.190

Zhao Gang, Song Mingbao, Huang Lan, Huang Lan. The Institute of Cardiovascular Disease of PLA, Xinqiao hospital, Third Military Medical University, 400037, China

Objectives Diabetes mellitus can accelerate the atherosclerotic process, but its mechanism remains unclear.

Methods to explore the effect of macrophages on the biological functions of EPCs under high glucose condition and the role of toll-like receptor 4 .

Methods and materials mouse peritoneal macrophages and mouse bone marrow-derived endothelial progenitor cells (EPCs) were cultured in media supplemented with high D-glucose $(450 \mathrm{mg} / \mathrm{dl}$ D-glucose) and $350 \mathrm{mg} / \mathrm{dl}$ L-glucose. Macrophages and EPCs were co-cultured in a transwell co-culture system. 3H-TdR incorporation, transwell and nitrate recovery were used respectively to measure the abilities of EPCs to proliferate, migrate and secrete nitric oxide (NO). In vitro tube-formation analysis also was used to evaluate the antigenic ability of EPCs. TLR 4 of macrophages was knocked-down with TLR 4 siRNA, and EPCs functions were analysed as above.

Results In the co-culture system, the 3H-TdR incorporation in EPC group was significantly higher than that in MC/EPC group (26070 $\pm 1969 \mathrm{cpm} /$ well vs $12200 \pm 1420 \mathrm{cpm} /$ well, $\mathrm{p}<0.01)$. The number of migratory EPC was $189.2 \pm 23.6$ and $97.8+13.6$ in EPC and MC/ EPC group respectively, and there was significant difference between two group $\mathrm{p}<0.01)$. Secreting $\mathrm{NO}$ in EPC group was superior to that in MC/EPC group. $12 \mathrm{~h}$ after cultured on EC Matrix, the values of in vitro tube-formation analysis in EPC group were significantly higher than those in MC/EPC group. The abilities of EPCs to proliferate, migrate, secrete $\mathrm{NO}$, and form tube in vitro in MC/EPC group were improved after the knockdown of TLR 4 in macrophages.

Conclusions under high glucose conditions, macrophage could impair the biological functions of EPCs, and TLR 4 of macrophages may play a key role in this process, which may be an important mechanism that diabetes mellitus accelerate the atherosclerotic process. 